\title{
ISOLATED POINTS IN THE BRANCH LOCUS OF THE MODULI SPACE OF COMPACT RIEMANN SURFACES
}

\author{
Ravi S. Kulkarni
}

\section{Introduction}

Let $g$ be an integer at least 2 and let $\mathcal{M}_{g}$ denote the moduli space of compact Riemann surfaces of genus $g$. Each of these Riemann surfaces has a canonical hyperbolic metric. All geometric notions will be tacitly with respect to this metric. For the generalities on these spaces cf. $[\mathrm{Be}],[\mathrm{H}]_{2}$. For $g \geq 3$ set

$$
\mathcal{B}_{g}=\left\{X \in \mathcal{M}_{g} \mid \text { Aut } X \neq e\right\}
$$

This is called the branch locus of $\mathcal{M}_{g}$. This is not a good definition for $g=2$, since every surface of genus 2 admits a hyperelliptic involution. So in this case set

$$
\mathcal{B}_{2}=\left\{X \in \mathcal{M}_{2} \mid \text { Aut } X \not \approx Z_{2}\right\} \text {. }
$$

It is known that $\mathcal{M}_{g}$ is a normal quasiprojective complex algebraic variety and is nonsingular outside $\mathcal{B}_{g}$. The geometric structure of $\mathcal{B}_{g}$ is of substantial interest in many contexts. In this note we shall consider the isolated points of $\mathcal{B}_{g}$.

We show that $\mathcal{B}_{g}$ contains isolated points only if $2 g+1$ is a prime and the converse also holds if this prime is $\neq 7$. Let $q=2 g+1$ be a prime. It turns out that if $X$ is an isolated point in $\mathcal{B}_{g}$ and $q=2 g+1$ is a prime then Aut $X \approx Z_{q}$ if $g \geq 3$, and $\approx Z_{10}$ if $g=2$. Let $G=$ Aut $X$ if $g \geq 3$, and a subgroup of Aut $X$ isomorphic to $Z_{5}$ if $g=2$. Then $X / G$ must have genus 0 with three branch points each of index $q$. A main point is to determine which $X$ in $\mathcal{M}_{g}$ with this type of structure are actually isolated points in $\mathcal{B}_{g}$. Fix a generator $u$ of $G$. Let $p: X \mapsto X / G$ be the canonical projection. The set $\mathcal{S}$ consisting of those $x$ in $X$ with $G_{x} \neq e$ is called the singular set and $\mathcal{B}=p(\mathcal{S})$ the branch set of the $G$-action. Now $u$ acts as a rotation at each point $x$ in $\mathcal{S}$ by an angle $2 r \pi / q$ where $1 \leq r \leq q-1$. The number $r$ depends on the choice of $u$ but since $G$ is abelian it is constant on the $G$-orbit of $x$. Thus from the three orbits of $G$ in $\mathcal{S}$ we can construct a characteristic symbol $[r, s, t]$ of the $G$-action, cf. Section 2 for a precise definition. The necessary and sufficient conditions for $X$

Partially supported by an NSF grant, and a PSC-CUNY award. 
to be an isolated point of $\mathcal{B}_{g}$ are given in terms of the characteristic symbols, cf. Theorem 3.6. Finally we show that the number of isolated points in $\mathcal{B}_{g}$ is 1 if $g=2,[(g-2) / 3]$ if $2 g+1$ is a prime $\neq 7$, and 0 otherwise. Here for a real number $x,[x]$ denotes the greatest integer $\leq x$.

In Section 4 we construct hyperbolic-geometric models of these surfaces representing isolated branch points. Actually we describe models for all cyclic group actions with fixed points so that the quotient has genus 0 and three branch points. These models are built out of hyperbolic polygons by identification of its sides in pairs. Among these are included the models for the famous surface of genus 2 admitting a group of order 48 and the Klein's surface of genus 3 admitting a group of order 168. Fricke and Klein, cf. [FK], p. 280 exhibit in principle a model for the latter surface which seems to have been reproduced at several places in the literature, cf. also Burnside [Bu], p. 420, and [RL]. We believe that the models constructed here are new and substantially simpler than the ones which have appeared in the literature. The details of the underlying topological idea in these constructions are given in Theorem 1 of $[\mathrm{K}]$ and works for any cyclic group action with a fixed point. It could be profitably used in some other contexts.

\section{Preliminaries}

2.1. Suppose $g \geq 3$. Let $X$ denote an isolated point in $\mathcal{B}_{g}$ and let $G=$ Aut $X$. In $[\mathrm{H}]_{2}$ it is described how a topological type of an orientation-preserving action of a finite group $H$ on a compact oriented surface of genus $g$ leads to a subvariety $\mathcal{V}_{H}$ in $\mathcal{B}_{g}$. That description shows that the dimension of $\mathcal{V}_{H}$ is positive if either the orbit space has positive genus or it is of genus 0 with at least 4 branch points. It follows that in our case for every nonidentity subgroup $H$ of $G$ we must have $X / H \approx$ the Riemann sphere with three branch points. Let $H$ be a subgroup of order $q$ where $q$ is some prime divisor of $|G|$. The branching indices of the action must be $q$. So by the Riemann-Hurwitz formula it follows that

$$
2 g-2=q\left\{1-\frac{1}{q}-\frac{1}{q}-\frac{1}{q}\right\}
$$

Or $q=2 g+1$. So first of all $2 g+1$ must be a prime, and if this prime is denoted by $q$ then $q$ is the only prime divisor of $|G|$. In other words $G$ must be a $q$-group.

2.2. Suppose if possible that $\mathrm{G} \not \approx Z_{q}$. Then being a $q$-group $G$ must contain a subgroup $H$ isomorphic either to $Z_{q} \times Z_{q}$ or to $Z_{q^{2}}$. In the first case the Riemann-Hurwitz formula applied to the $H$-action would show that

$$
2 g-2=q^{2}\left\{1-\frac{1}{q}-\frac{1}{q}-\frac{1}{q}\right\},
$$

which is inconsistent with $q=2 g+1$. In the second case since $Z_{q^{2}}$ is cyclic at least two of the branching indices would have to be $q^{2}$ and the third may be $q$ or 
$q^{2}$, cf. $[\mathrm{H}]_{1}$. In either case one easily checks that this data is inconsistent with $q=2 g+1$. So $G \approx Z_{q}$.

2.3. Let $p: X \mapsto X / G$ be the canonical projection, $\mathcal{S}$ the singular set and $\mathcal{B}=\{A, B, C\}$ the branch set of the $G$-action. Fix a generator $u$ of $G$. Since $G$ is abelian it is clear that at each point in a $G$-orbit in $\mathcal{S}$ the action of $u$ in a small neighborhood is by a rotation through an angle which is a fixed multiple of $2 \pi / q$. Let $a, b, c$ be these multiples determined $\bmod q$ over $p^{-1}(A), p^{-1}(B), p^{-1}(C)$, respectively. It follows by the Hurwitz's theory that

$$
a+b+c \equiv 0 \quad \bmod q .
$$

Also

each of $a, b, c$ is $\not \equiv 0 \quad \bmod q$.

Note that changing a generator $u$ by some power $\lambda$ of it replaces $a, b, c$ by $\lambda a$, $\lambda b, \lambda c$ where $\lambda$ is $\not \equiv 0 \bmod q$.

2.4. Let $N$ denote the set of unordered triples $\{r, s, t\}$ of numbers counted $\bmod q$ such that each is $\not \equiv 0 \bmod q$ and $r+s+t \equiv 0 \bmod q$. Now the multiplicative group $Z_{q}^{*}=Z_{q}-\{0\}$ acts on $N$ by componentwise multiplication. An orbit of $\{r, s, t\}$ under this action will be denoted by $[r, s, t]$. A standard consequence from Hurwitz's general theory in this setup is that there is a canonical surjective map from $N / Z_{q}^{*}$ onto the set of all Riemann surfaces admitting a $Z_{q}$-action with quotient the Riemann sphere with 3 branch points. It is a special case of a wellknown theorem of Nielsen on cyclic group actions on compact orientable surfaces that in our setup this map is actually a bijection, cf. $[\mathrm{N}],[\mathrm{Sm}],[\mathrm{E}]_{1,2}$, cf. also $[\mathrm{H}]_{2}$ Theorem 14 where however a different equivalence relation is used. (In terms of Fuchsian groups this amounts to the fact that there is a bijection of $N / Z_{q}^{*}$ onto the set of $\mathrm{PSL}_{2}(R)$-conjugacy classes of normal subgroups $\approx \pi_{1}(X)$ of the triangle group $\Delta=\Delta_{\{q, q, q\}}$ with quotient $\approx Z_{q}$.) Consequently we can associate symbols $[r, s, t]$ in a one-to-one manner to the Riemann surfaces admitting a $Z_{q}$-action with quotient of genus 0 with 3 branch points. A symbol of this type will be called the characteristic symbol of the corresponding Riemann surface.

2.5. The following consequence of a crucial theorem of Singerman, cf. [S] Theorems 1 and 2, will also be used in the sequel. The only Fuchsian groups which contain the triangle group $\Delta_{\{q, q, q\}}$, up to conjugacy in $\mathrm{PSL}_{2}(\mathbf{R})$, are the triangle groups $\Delta_{\{2, q, 2 q\}}, \Delta_{\{3,3, q\}}$ and $\Delta_{\{2,3,2 q\}}$, containing $\Delta_{\{q, q, q\}}$ as a subgroup of index $2,3,6$ respectively, except for $q=7$ in which case in addition to the above inclusions $\Delta_{\{7,7,7\}}$ is also contained in $\Delta_{\{2,3,7\}}$ as a subgroup of index 24 . 


\section{Isolated points of $\mathcal{B}_{g}$}

3.1. We shall now derive necessary and sufficient conditions for a Riemann surface to be an isolated point in $\mathcal{B}_{g}$. First

Lemma. Let $g \geq 3$. Let $G \approx Z_{q}, q=2 g+1$ a prime, and let $X$ be a compact Riemann surface of genus $g$ admitting a $Z_{q}$-action with quotient of genus 0 with 3 branch points. Let $[r, s, t]$ be the characteristic symbol of $X$. If $X$ is an isolated point in $\mathcal{B}_{g}$ then no two of $r, s, t$ can be equal.

(Note: Although the individual values of $r, s, t$ in a characteristic symbol are defined only up to an action by Aut $Z_{q}$ the notion that two of $r, s, t$ are equal or not is welldefined.)

Proof. It is known that (even for $q$ not necessarily a prime) there is a unique compact Riemann surface $Y$ of genus $g \geq 2$ admitting an action of a cyclic group $A$ of order $2 q=4 g+2$. It is hyperelliptic with equation $w^{2}=z^{2 g+1}-1$, cf. [K]. Moreover $Y / A$ is the Riemann sphere with three branch points with branching indices $\{2, q, 2 q\}$. Let $p: Y \mapsto Y / A$ be the canonical projection. The two points in $Y$ lying over the branch point of index $q$ and the one lying over the branch point of index $2 q$ are fixed by the subgroup $B \approx Z_{q}$ of $A$. It is easy to see from the Riemann-Hurwitz formula that $Y / B$ is the Riemann sphere with three branch points. Since the two points in $Y$ lying over the branch point of index $q$ in $Y / A$ are permuted by the element of order 2 in $A$ it is clear that the characteristic symbol of $Y$ with respect to the $B$-action has the form $[r, r, t]$. Notice also that there is only one characteristic symbol $[r, s, t]$ with two of $r, s, t$ equal. So by Nielsen's theorem $Y$ is the only Riemann surface up to biholomorphic equivalence which admits a $\mathrm{Z}_{q}$-action with the characteristic symbol $[r, r, t]$. But by construction Aut $Y$ has at least $4 g+2$ elements. So by (2.2) $Y$ cannot be an isolated point in $\mathcal{B}_{g}$

3.2. Lemma. Let $G, X,[r, s, t]$ be as in Lemma (3.1). Fix a generator $u$ of $G$, and w.r.t. $u$ let $\{r, s, t\}$ be an element in $N$ (cf. (2.4)) which represents $[r, s, t]$. If $X$ is an isolated point in $\mathcal{B}_{g}$ then the following holds: If $\{r, s, t\}=$ $\{\lambda r, \lambda s, \lambda t\}$ for $\lambda$ in $Z_{q}^{*}$ then $\lambda=1$. If $q$ is congruent to $-1 \bmod 3$ then this condition is automatic. If $q$ is congruent to $1 \bmod 3$ and $\{r, s, t\}=\{\lambda r, \lambda s, \lambda t\}$ for $\lambda \neq 1$ then $\lambda$ is a cubic root of unity and $[r, s, t]=\left[1, \lambda, \lambda^{2}\right]$

Proof. Suppose we have $\{r, s, t\}=\{\lambda r, \lambda s, \lambda t\}$ for $\lambda \neq 1$. This means that as unordered triples of elements in $Z_{q}$ the two triples are equal. So we may take $\lambda r=s, \lambda s=t, \lambda t=r$. So $\lambda^{3} r=r$ or $\lambda^{3}=1$. In particular the multiplicative group $Z_{q}^{*}$ contains an element of order 3 . So $q$ must be congruent to $1 \bmod 3$. This proves the last statement in the lemma.

Now assuming $q$ to be congruent to $1 \bmod 3$ we form the group $H=Z_{q} \rtimes Z_{3}$, i.e. the semidirect product of $Z_{q}$ by $Z_{3}$ where $Z_{3}$ acts nontrivially on $Z_{q}$. If $\lambda \neq 1$ 
is a cube root of unity $\bmod q$ then a presentation of $H$ may be taken as

$$
H=\left\langle x, y \mid x^{q}=y^{3}=e, y x y^{-1}=x^{\lambda}\right\rangle .
$$

In this group $x y$ has order 3 . There is an obvious map of the triangle group $\Delta_{3,3, q}$ onto $H$ with a torsionfree kernel. Correspondingly we get an action of $H$ on a Riemann surface $Y$ such that $Y / H$ has genus 0 with three branch points with branching indices $3,3, q$. If $h$ is the genus of $Y$ the Riemann-Hurwitz formula gives

$$
2 h-2=3 q\left\{1-\frac{1}{3}-\frac{1}{3}-\frac{1}{q}\right\} .
$$

This implies that $h=g$. So we have an $H$-action on a Riemann surface of genus $g$. Let $K=\langle x\rangle$. Over the branch point of index $q$ in $Y / H$ there are three points in $Y$ which are all fixed by $K$ since $G$ is normal in $H$. Moreover the intersection of $K$ with the stabilizers of all points in $Y$ lying over the branch points of index 3 must be $\{e\}$ since 3 and $q$ are relatively prime. It follows that the $K$-action has exactly 3 branch points. Another application of the RiemannHurwitz formula shows that $Y / K$ has genus 0 . Now the presentation (3.2.1) of $H$ clearly shows that the characteristic symbol of this $K$-action is $\left[1, \lambda, \lambda^{2}\right]$. By construction Aut $Y$ has at least $3 q$ elements, so $Y$ is not an isolated point of $\mathcal{B}_{g}$. An appeal to Nielsen's theorem shows that $Y$ is the only element of $\mathcal{B}_{g}$ which has the characteristic symbol $\left[1, \lambda, \lambda^{2}\right]$. So the lemma follows.

3.3. Now let $g, q, G, X$ be as in Lemma (3.1) and let $[r, s, t]$ be the characteristic symbol of $X$. Suppose that no two of $r, s, t$ are equal and if $q \equiv$ $1, \bmod 3$ then $[r, s, t] \neq\left[1, \lambda, \lambda^{2}\right]$ where $\lambda \neq 1$ is a cubic root of unity $\bmod q$. We claim that $X$ must be an isolated point in $\mathcal{B}_{g}$. Suppose not. Then $G$ is a proper subgroup of $H=$ Aut $X$. Correspondingly we have a proper inclusion of $\Delta_{\{q, q, q\}}$ (which uniformizes the $G$-action) in some Fuchsian group $\Delta_{H}$ (which uniformizes the $H$-action) as a subgroup of index $(H: G)$. Now the possibilities for $\Delta_{H}$ are classified by Singerman, cf. (2.5). Among these possibilities one need not consider $\Delta_{\{2,3,2 q\}}$, since this inclusion factors through the inclusions in $\Delta_{\{2, q, 2 q\}}$, or $\Delta_{\{3,3, q\}}$. Similarly for $q=7$ one need not consider the inclusions in $\Delta_{\{2,3,7\}}$, since this inclusion factors through an inclusion in $\Delta_{\{3,3,7\}}$. Now the inclusions in $\Delta_{\{2, q, 2 q\}}$, or $\Delta_{\{3,3, q\}}$ are of index 2 and 3 respectively. In the first case $H$ would be cyclic since $2 q$ is one of the branching indices, and we are exactly in the situation encountered in the proof of Lemma (3.1). We saw there that in this case two of $r, s, t$ are equal. In the second case $H$ is a group of order $3 q$. By Sylow's theorem one sees that there is only one $q$-Sylow subgroup of $H$ and so it is normal, and $H$ is isomorphic to a semidirect product of $Z_{q}$ by $Z_{3}$. If $Z_{3}$ acts trivially on $Z_{q}$ then $H$ would be cyclic and this would be inconsistent with the branching $\{3,3,7\}$. (In a cyclic group action with quotient of genus 0 and $k$ branching indices the l.c.m. of any $k-1$ branching indices equals the order of the 
group.) Since Aut $Z_{q}$ contains a unique subgroup of order 3 it follows that $H$ is uniquely determined up to isomorphism. It is as given by the presentation (3.2.1) and we are exactly in the situation encountered in the proof of Lemma (3.2). As we saw there in this case the characteristic symbol would be $\left[1, \lambda, \lambda^{2}\right]$ where $\lambda \neq 1$ is a cubic root of unity $\bmod q$. These contradictions show that $X$ must be an isolated point in $\mathcal{B}_{g}$.

3.4. The case of genus $g=2$ needs a special consideration in view of the different definition of $\mathcal{B}_{2}$, cf. Section 1. If $X$ is an isolated point in $\mathcal{B}_{2}$ then for every subgroup $H$ of Aut $X, H \neq\langle$ the hyperelliptic involution 〉 we have $X / H$ of genus 0 with three branch points. If the order of $H$ is divisible by a prime $q \neq 2$ then as in (2.1) $q$ must be 5. As in (3.1), it then follows that Aut $X$ contains a subgroup isomorphic to $Z_{10}$ and $X$ is the hyperelliptic surface $w^{2}=z^{5}-1$. From $[\mathrm{K}]$, Theorem 3 it follows that in this case Aut $X$ is not larger and $X$ is indeed an isolated point in $\mathcal{B}_{2}$. On the other hand if Aut $X$ is a 2 -group of order at least 4 then it contains a subgroup isomorphic to $Z_{2} \times Z_{2}$ or $Z_{4}$. In either case by Riemann-Hurwitz formula it already follows that for some subgroup $H \neq\langle$ the hyperelliptic involution $>X / H$ is not of genus 0 with 3 branch points, and so $X$ is not isolated.

3.5. Now for $g \geq 3$ we compute the number of isolated points in $\mathcal{B}_{g}$. We have already observed that if this number is nonzero then $q=2 g+1$ is a prime. Moreover if $X$ is isolated then Aut $X$ is isomorphic to $Z_{q}$ and the characteristic symbol $[r, s, t]$ of $X$ satisfies

i) None of $r, s, t$ is 0 , and their sum is 0 .

ii) No two of $r, s, t$ are equal.

iii) If $q \equiv 1 \bmod 3$, and $\lambda$ is a cubic root of unity $\bmod q$ then $[r, s, t] \neq\left[1, \lambda, \lambda^{2}\right]$.

So we need to compute the number of the characteristic symbols satisfying these conditions. Now in the triples $(r, s, t)$ satisfying i) there are $q-1$ choices for $r$. With $r$ fixed $s$ cannot be 0 nor $-r$, for the latter would imply $t=0$. So there are $q-2$ choices for $s$. Now $t$ is fixed. So the number of such triples is $(q-1)(q-2)$. Now the condition ii) further removes $3(q-1)$ triples of the form $(r, r,-2 r),(r,-2 r, r),(-2 r, r, r)$ leaving the number $(q-1)(q-5)$ of triples. Since the components in these triples are pairwise distinct it follows that there are $(q-1)(q-5) / 6$ corresponding unordered triples. Now consider the $Z_{q}^{*}$-action by componentwise multiplication on this set. If $q \not \equiv 1 \bmod 3$ this action is free and so there are $(q-5) / 6$ orbits which is the required number of characteristic symbols in this case. If $q \equiv 1 \bmod 3$ and $\lambda$ is a cubic root of unity $\bmod q$ then the action is free outside the orbit of $\left[1, \lambda, \lambda^{2}\right]$ which contains $(q-1) / 3$ elements. So the required number of characteristic symbols in this case is $((q-5) / 6)-(1 / 3)=(q-7) / 6$. In either case one sees that there are $[(g-2) / 3]$ characteristic symbols.

Summarizing we have proved the following theorem.

3.6. Theorem. The number of isolated points in $\mathcal{B}_{g}$ is 1 if $g=2,[(g-2) / 3]$ 
if $q=2 g+1$ is a prime $>7$, and 0 otherwise. The isolated point in $\mathcal{B}_{2}$ is the hyperelliptic surface $w^{2}=z^{5}-1$. For $g \geq 5$ the isolated Riemann surfaces are precisely those $X$ 's for which Aut $X$ is isomorphic to $Z_{q}$ and such that in their characteristic symbols $[r, s, t]$ no two of $r, s, t$ are equal and if $q \equiv 1 \bmod 3$, and $\lambda$ is a cubic root of unity $\bmod q$ then $[r, s, t] \neq\left[1, \lambda, \lambda^{2}\right]$.

3.7. Examples. i) For $g=3$ the only characteristic symbols are $[1,1,5]$ and $[1,2,4]$. The latter does not correspond to an isolated point since $2^{3}=1 \bmod 7$, and by (3.2) nor does the first. So there are no isolated points in $\mathcal{B}_{3}$.

ii) For $g=5$ the characteristic symbols are $[1,1,9]$ and $[1,2,8]$. The first does not correspond to an isolated point by (3.2), the second does. So there is exactly one isolated point in $\mathcal{B}_{5}$.

ii) For $g=6$ the characteristic symbols are $[1,1,11],[1,2,10]$, [1,3,9]. Only the second corresponds to an isolated point.

\section{Geometric models}

4.1. In this section we construct a model for a Riemann surface $X_{g}$ admitting a $Z_{l}$-action with a fixed point and with quotient of genus 0 with three branch points. We may take its characteristic symbol in the form $[1, a, b]$. We fix a generator $x$ of $Z_{l}$ so that on each of the points of the three orbits of singular points $x, x^{a}, x^{b}$ act by a rotation by an angle $(2 \pi) / l,(2 \pi) / u,(2 \pi) / v$ respectively. Then the order of $x^{a}$, respectively $x^{b}$ is $u=l /(l, a)$, respectively $v=l /(l, b)$. Notice that l.c.m. $(u, v)$ must be $l$, and $1+a+b \equiv 0 \bmod l$. Also $2 g-2=$ $l\left\{1-u^{-1}-v^{-1}-l^{-1}\right\}$.

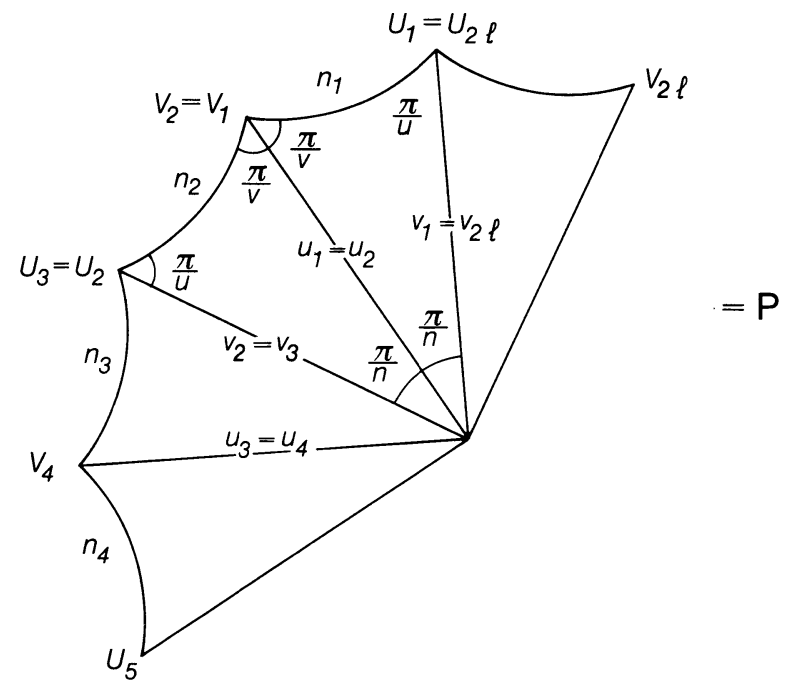

4.2. Let $T$ be a hyperbolic triangle with angles $\pi / l, \pi / u, \pi / v$. Take $2 l$ copies $T_{1}, T_{2}, \ldots T_{2 l}$. The vertices of $T_{i}$ are $N_{i}, U_{i}, V_{i}$, with angles $\pi / l, \pi / u$, 
$\pi / v$ respectively and their opposite edges are denoted by $n_{i}, u_{i}, v_{i}$, respectively. The polygon $P$ is obtained from the union of the $T_{i}$ 's by identifying all $N_{i}$ 's to a single vertex $N_{0}$, and further identifying $u_{2 i-1}$ with $u_{2 i}$, and $v_{2 i}$ with $v_{2 i+1}$, where the subscripts are counted $\bmod 2 l$. The sides of $P$ are $n_{1}, n_{2}, \ldots, n_{2 l}$. We also have the identification of the vertices $U_{2 i}$ with $U_{2 i+1}$, and $V_{2 i-1}$ with $V_{2 i}$. For convenience we may take the vertices of $P$ as $U_{1}, V_{2}, U_{3}, V_{4}, \ldots, V_{2 l}$.

4.3. Let the generator $x$ of $Z_{l}$ act on $P$ as a rotation through an angle $2 \pi / l$ fixing the center $N_{0}$. On the sides of $P$ it induces the permutation $n_{i} \mapsto n_{i+2}$ and on the vertices $U_{i} \mapsto U_{i+2}$ and $V_{i} \mapsto V_{i+2}$. Now consider the sidepairing $\sigma$ : $n_{2 i-1} \mapsto n_{2 i+2 a}$, and let $X$ denote the surface obtained by isometrically identifying $n_{2 i-1}$ with $n_{2 i+2 a}$ in an orientation-reversing manner. Since $x$ commutes with $\sigma$ we obtain a $Z_{l}$-action on $X$. Since $P / Z_{l}$ is clearly isometric to the space obtained from $T_{1} \cup T_{2}$ by glueing $u_{1}$ with $u_{2}$ and $v_{1}$ with $v_{2}$ it is clear that $X / Z_{l}$ is isometric to the space obtained from $T_{1} \cup T_{2}$ by glueing $u_{1}$ with $u_{2}, v_{1}$ with $v_{2}$, and $n_{1}$ with $n_{2}$, i.e. $X / Z_{l}$ has genus 0 and three branch points with branching indices $u, v, l$. In particular the genus of $X$ is determined from the equation $2 g-2=l\left\{1-u^{-1}-v^{-1}-l^{-1}\right\}$.

4.4. Now we claim that the characteristic symbol of this $Z_{l}$-action on $X$ is $[1, a, b]$. It is convenient to do a computation on $P$ and interpret it in $X$. For any point or subset in $P$ we shall denote its image in $X$ by a bar overhead. Consider a small arc $\alpha$ starting from $V_{2}$ lying in $u_{2}$ and oriented towards $N_{0}$. See the figure below. Since $n_{1}$ is identified with $n_{2 a+2}$ we see that $\bar{V}_{2}=\bar{V}_{2 a+2}$. Also $x^{a}(\alpha)$ is an arc starting from $V_{2 a+2}$ lying in $u_{2 a+2}$ and oriented towards $N_{0}$. It is easy to see that the angle between $\bar{\alpha}$ and $x^{a}(\bar{\alpha})$ is $2 \pi / v$. In other words on $X$ we see $x^{a}$ fixing $\bar{V}_{2}$ and inducing in its neighborhood a rotation through $2 \pi / v$. Similarly it is seen that $x^{a+1}$ fixes $\bar{U}_{1}$ and induces there a rotation through $-2 \pi / u$. So $x^{-a-1}=x^{b}$ fixes $\bar{U}_{1}$ and induces there a rotation through $2 \pi / u$. This shows that the characteristic symbol of this action is $[1, a, b]$. By the arguments in the previous section $X$ is the unique Riemann surface admitting a $Z_{l}$-action with quotient of genus 0 with three branch points and with the characteristic symbol $[1, a, b]$. 
4.5. We describe three examples of the above procedure. First as noted in (3.7) there is a unique isolated branch point in $\mathcal{B}_{5}$. It admits a $Z_{11}$-action with the characteristic symbol $[1,2,8]$. The corresponding Riemann surface may be represented by a hyperbolic 22 -gon with all the internal angles $2 \pi / 11$ so that if its sides are cyclically labelled $n_{1}, \ldots, n_{22}$ then the sidepairing is $n_{2 i-1} \mapsto n_{2 i+4}$.

Next we describe the famous surface of genus 2 which admits a group of order 48. This group is isomorphic to $\mathrm{Gl}_{2}\left(Z_{3}\right)$, i. e. the group of all nonsingular $2 \times 2$ matrices with entries in a field with 3 elements. It contains an element of order 8 . The Riemann-Hurwitz formula easily implies that the $Z_{8}$-action on a surface of genus 2 must have the quotient of genus 0 with branching indices $2,8,8$. It is a fortuitous circumstance in this case that this data uniquely determines the characteristic symbol. It is $[1,4,3]$. So the surface is completely determined by the fact that it is of genus 2 and that it admits a $Z_{8}$-action! By the above procedure it is obtained from a 16 -gon whose internal angles are alternately $2 \pi / 8$ and $\pi$. The sidepairing is $n_{2 i-1} \mapsto n_{2 i+8}$. It is easy to see that if we agree not to count the vertices with angle $\pi$ as vertices then we have an octagon with all the internal angles $2 \pi / 8$, and the sidepairing is a standard one, namely $a b c d a^{-1} b^{-1} c^{-1} d^{-1}$ !

Finally we describe the famous Klein's surface $X$ of genus 3 which admits a group of order 168. This group is isomorphic to $\mathrm{PSL}_{2}\left(Z_{7}\right)$. It contains a cyclic subgroup of order 7 which is unique up to conjugacy in $\mathrm{PSL}_{2}\left(Z_{7}\right)$. Again it is easy to see that the Riemann-Hurwitz formula implies that the $Z_{7}$-action on a surface of genus 3 must have the quotient of genus 0 with branching indices $7,7,7$. There are however two possibilities for the characteristic symbol, namely $[1,1,5]$ and $[1,2,4]$. Which is the possibility for the Klein's surface? To see this fix a cyclic subgroup $A$ of order 7 in $\mathrm{PSL}_{2}\left(Z_{7}\right)$. Its normalizer $N$ is a nonabelian group of order 21. There is a unique nonabelian group of order 21 up to isomorphism, namely

$$
\left\langle x, y \mid x^{7}=y^{3}=e, y x y^{-1}=x^{2}\right\rangle .
$$

Since $N$ leaves the fixed point set of $A$ in $X$ invariant the above presentation shows that the characteristic symbol must be $[1,2,4]$. So by the above procedure a model for $X$ may be obtained from a hyperbolic 14 -gon with all internal angles $2 \pi / 7$ where the sidepairing is given by $n_{2 i-1} \mapsto n_{2 i+4}$. 

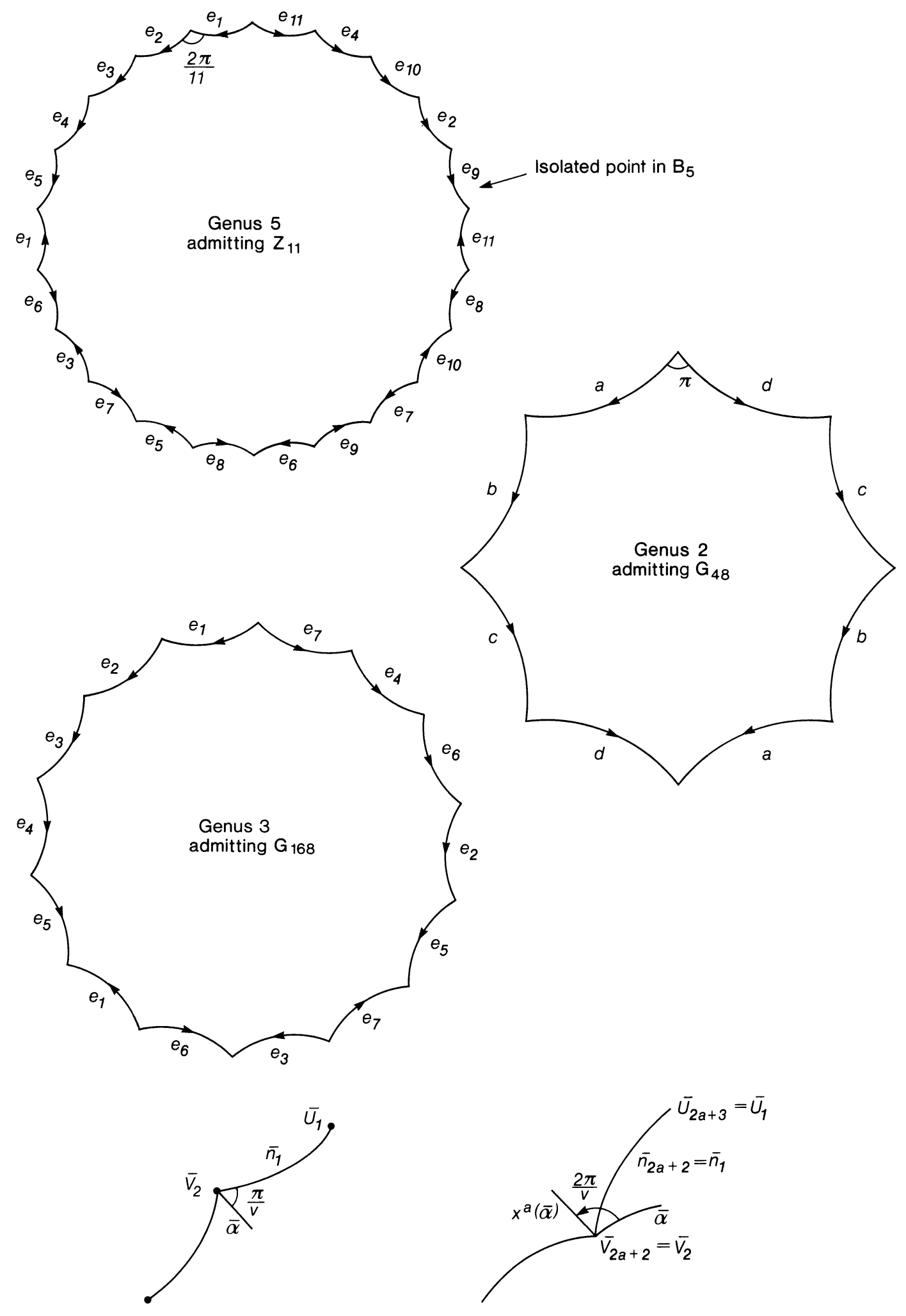


\section{References}

[Be] Bers, L.: Uniformization, moduli, and Kleinian groups. - Bull. London Math. Soc. 4, 1972, 257-300.

[Bu] Burnside, W.: The theory of groups. - Cambridge University Press, Cambridge, 1911 (second edition).

$[E]_{1,2}$ Edmonds, A.: Surface symmetry I and II. - Michigan Math. J. 29, 1982, 171-183; ibid. $30,1983,143-154$.

[FK] FrICkE, R., and F. KLEIN: Theorie der Automorphen Funktionen. - Teubner, Leipzig, 1901.

$[\mathrm{H}]_{1}$ HARVEY, J.W.: Cyclic groups of automorphisms of a compact Riemann surface. - Quarterly J. Math. 17, 1966, 86-97.

$[\mathrm{H}]_{2}$ HaRveY, J.W.: On branch loci in Teichmüller space. - Trans. Amer. Math. Soc. 153, 1971, 387-399.

[Hu] Hurwitz, A.: Über algebraische Gebilde mit eindeutigen Transformationen in sich.. Math. Ann. 41, 1893, 403-442.

[K] KulKarni, R.S.: Riemann surfaces admitting large automorphism groups. - Preprint.

[N] Nielsen J.: Die Struktur periodischer Transformationen von Flächen. - Danske Vid. Selsk. Mat.-Fys. Medd. 15, 1937, 1-77.

[RL] RAUCH, H.E., and J. LEwitTES: The Riemann surface of Klein with 168 automorphisms. - Problems in Analysis, a Symposium in Honor of Solomon Bochner, Princeton University Press, Princeton, N.J., 1970, 297-308.

[S] Singerman D.: Finitely maximal Fuchsian groups. - J. London Math. Soc. (2) 6, 1972, 29-38.

[Sm] Smith, P.A.: Abelian actions on 2-manifolds. - Michigan Math. J. 14, 1967, 257-275.

[W] Wiman, A.: Über die hyperelliptischen Curven und diejenigen von Geschlechte $p=3$ welche eindeutige Transformationen in sich zulassen. - Bihang till K. Svenska Vet.Akad. Handlingar, Stockholm, 1895-6, bd.21, 1-23.

City University of New York

Graduate Center

Department of Mathematics

33, W. 42nd Street

New York, N.Y. 10036

U.S.A.

Received 15 December 1989 